\title{
Mieux comprendre le rôle des neurotrophines dans le système nerveux grâce à l'invalidation génique
}

Les facteurs de la famille du nerve growth factor (NGF), les neurotrophines, sont actuellement les plus étudiées des molécules à activité neurotrophique $\left(\mathrm{m} / \mathrm{s} n^{\circ} 7\right.$, vol. $6, p .700$; $n^{\circ} 6$, vol. 7, p. 630). Cet engouement est le fait de deux événements simultanés. D'une part, la mise en évidence de l'existence de molécules à structure proche de celle du NGF, qui englobent, dans l'ordre chronologique de leur découverte, le brainderived neurotrophic factor (BDNF) et les neurotrophines-3 (NT-3), - $4 / 5$ (NT-4/5) et $-6(\mathrm{NT}-6)\left(\mathrm{m} / \mathrm{s} n^{\circ} 1\right.$, vol. 11, p. 145), voir [1]). D'autre part, l'identification des récepteurs à forte affinité des neurotrophines comme appartenant à une famille de récepteurs à activité tyrosine kinase du même type qu'une oncoprotéine initialement détectée dans un cancer du colon, les Trk (tropomyosin receptor kinase ( $m / s{ }^{\circ} 6$, vol. 7, p. 620) (voir $[2,3])$. La diversité des protéines et de leurs récepteurs a soudainement bousculé le paysage de l'unique NGF et de son récepteur à faible affinité, redessinant et élargissant avec une surprenante rapidité la carte de leurs implications physiopathologiques.

Cette rapidité est due en grande partie au développement des techniques de biologie moléculaire. Pour en suivre la chronologie (Tableau I), le BDNF a été caractérisé par une longue et fastidieuse purification à partir d'extraits de cerveaux de porc [4]. Les autres neurotrophines (NT3 , NT-4/5 et NT-6) ont, en revanche, été caractérisées par comparaison de séquences dans des banques, en prenant appui sur la conservation des régions entourant les cystéines, observée entre le NGF et le BDNF. Faute d'anticorps spécifiques, la localisation des facteurs a été en grande partie assurée par des techniques d'hybridation in situ. Ces techniques ont également permis d'observer les variations des contenus en $\mathrm{ARNm}$ dans différents modèles pathologiques, donnant naissance ainsi à quelques hypothèses fonctionnelles. Le clonage des ADNc codant pour les récepteurs à forte affinité a conduit à la caractérisation de multiples isoformes, dont les rôles restent à préciser. L'obtention de protéines recombinantes humaines pour le BDNF et les NT-3, - 4/5 et - 6 a pallié le manque d'une source suffisamment riche comme l'est la glande sous-maxillaire de souris pour le NGF et permis d'observer les répercussions d'un apport exogène de fac- teurs. La réalisation d'animaux transgéniques, porteurs d'invalidation des gènes des neurotrophines ou de leurs récepteurs, marque aujourd'hui une nouvelle étape dans la détermination de leurs fonctions respectives. Les résultats issus de ces différents événements ont progressivement remis en question plusieurs dogmes. Tout d'abord, la diversité des facteurs a redistribué des rôles fonctionnels trop multiples pour être le fait d'une seule molécule, limitant de ce fait ceux alloués au NGF. Ensuite, l'identification du domaine tyrosine kinase des récepteurs à forte affinité a permis de préciser la cascade d'événements intracellulaires induits par la liaison des neurotrophines. Cette identification a, en outre, considérablement réduit l'étendue du rôle du récepteur à basse affinité, qui, du

\section{Tableau I}

\section{LA SAGA DES NEUROTROPHINES}

1951 Découverte du NGF

1962 Purification et séquençage du NGF

1982 Purification du BDNF

1986 Séquençage du récepteur à faible affinité des neurotrophines

1989 Séquençage de l'ADNc du BDNF

1990 Identification de la NT-3

1991 Identification de la NT-4/5

1991 Identification des Trk

1992- Réalisation de souris transgéniques pour le NGF

1993- Réalisation des souris à invalidation dirigée des gènes de neurotrophines ou de leurs récepteurs

1995 Identification de la NT-6 
coup, est une énigme. Enfin, les exemples répétés de co-expression des gènes des récepteurs à forte affinité et de leurs ligands ont suggéré la prépondérance, au moins pour le BDNF, d'un nouveau mode d'action, paracrine ou autocrine $\left(\mathrm{m} / \mathrm{s} n^{\circ} 11\right.$, vol. 9, p. 1266), qui s'ajoute au mode prototypique d'action par transport rétrograde après capture par les terminaisons, démontré à l'origine pour le NGF. Cette hypothèse a été confirmée dernièrement par l'équipe de Lindsay [5].

La détermination des fonctions respectives des neurotrophines est à l'évidence le principal enjeu des études actuelles. Peu de véritables outils pharmacologiques, sinon aucun, sont disponibles pour approcher ces fonctions in vivo. A cause du manque de disponibilité des protéines et de leur faible immunogénicité, les célèbres expériences d' immunosympathectomie" effectuées grâce aux anticorps anti-NGF par Rita Levi-Montalcini (voir [6]) et qui ont démontré l'implication du facteur dans le développement des neurones périphériques, n'ont pu être répétées avec les autres neurotrophines. Quelques hypothèses d'implication dans la protection contre les atteintes excitotoxiques ont été suggérées grâce à l'élaboration de lignées, modifiées génétiquement pour produire et sécréter les facteurs, que l'on a greffées chez des animaux adultes. Ces lignées, bien qu'effectivement sécrétantes, présentent cependant différents problèmes, propres à la régulation du transgène ou liés à la transplantation, qui en ont rapidement limité l'intérêt. La majeure partie des données a donc été acquise en culture et concerne principalement l'implication des neurotrophines dans des mécanismes tardifs de la neurogenèse, tels que la différenciation et la mort neuronale liée au développement. Pourtant, la présence des ARNm du BDNF et de la NT-3 dès les premières étapes du développement embryonnaire évoque d'autres types d'implications et, là encore, les observations in vitro suggèrent des effets mitogènes ou différenciateurs de ces deux neurotrophines sur des précur-
Une nouvelle étape vient d'être franchie par les techniques d'invalidation génique dirigée contre l'un ou plusieurs des facteurs ou de leurs récepteurs [7]. Les souris portant une invalidation du gène de TrkA [trkA (-/-)], le récepteur à haute affinité du NGF, montrent des déficiences sensorielles durant la première semaine postnatale. Ces déficiences affectent la réactivité aux stimulus nociceptifs, olfactifs et tactiles, correspondant respectivement à une perte d'environ $70 \%$ des neurones trigéminés et des neurones sensoriels. Après quelques semaines, les animaux trkA (-/-) développent une symptomatologie particulière (pelade, ulcérations épidermiques, opacités cornéales, anomalies de différents organes), qui s'accentue avec l'âge. Le ganglion cervical supérieur, normal jusqu'à la naissance, dégénère rapidement dans les premières semaines postnatales. En revanche, les neurones cholinergiques centraux ne dégénèrent pas chez les souris trk $(-/-)$, même si l'innervation cholinergique de l'hippocampe et du cortex est réduite. Aucun dysfonctionnement moteur n'a été observé. Ces informations corroborent et complètent celles déjà connues. En accord avec de nombreux travaux précédents, le NGF seul est donc nécessaire à la survie de certaines populations de neurones sensoriels et sympathiques, mais pas de toutes, ce qui suggère qu'une autre neurotrophine, susceptible de se lier à TrkA, puisse suppléer son absence. Contrairement à ce qui a été longtemps suggéré, le NGF n'intervient pas dans la survie des neurones cholinergiques centraux, mais il est nécessaire au développement correct de leur arborisation axonale.

L'invalidation de $t r k \mathrm{~B}$ est létale dans la première semaine postnatale par suite d'une incapacité des nouveaunés de se nourrir. Les anomalies neurologiques que présentent les animaux trkB (-/-) incluent une perte neuronale dans les ganglions trigéminés et le noyau moteur du nerf facial, ainsi que dans les ganglions noueux et pétreux, qui sont impliqués respectivement dans la prise de nourriture et le relais d'informations sensorielles en provenance des viscères. Aucun défaut majeur n'a pour l'ins- tant été observé dans le système nerveux central de ces animaux, à l'exception d'une perte de $30 \%$ des motoneurones spinaux aux niveaux L2-L5. Ici encore, ces observations confirment les données déjà acquises, montrant une implication du BDNF dans la survie de populations neuronales périphériques recouvrant seulement partiellement celles sensibles au NGF, ainsi que d'une sous-population de motoneurones. En outre, elles révèlent que, de même que pour le NGF, le développement des autres structures centrales ne dépend pas de ce facteur. Les animaux portant une invalidation de $\operatorname{trk} C$ ont un développement postnatal normal. Ils présentent pourtant, peu après la naissance, des mouvements anormaux qui provoquent une posture incorrecte des membres postérieurs. Ces modifications posturales reflètent un défaut de la proprioception. Les animaux trkC (-/-) montrent en effet une perte importante des afférences musculaires de type Ia et une perte de certains neurones des ganglions des racines dorsales, probablement ceux qui donnent naissance à ces afférences.

Ces techniques d'invalidation génique ont deux limites. D'une part, l'absence continue de la molécule, depuis les premiers stades de développement, ne permet pas de mettre en évidence d'éventuelles évolutions de la sensibilité aux différents facteurs au cours de la vie d'une même population cellulaire. A ce propos, l'exemple du ganglion trigéminé est intéressant à rappeler. Ces neurones présentent un changement de sensibilité aux neurotrophines durant leur maturation. Dépendants du BDNF et des NT-3 et - 4/5 au moment de leur synaptogenèse, ils perdent cette sensibilité pour devenir, par la suite, dépendants du NGF. Ces neurones disparaissent donc systématiquement chez les souris portant des délétions des récepteurs à forte affinité, quels qu'ils soient. D'autre part, les études in vitro ont suggéré l'existence de mécanismes compensateurs des neurotrophines entre elles, que les invalidations géniques simples ne permettent pas d'observer. Différentes populations 
cellulaires portent plusieurs des récepteurs à forte affinité et leur spécificité pour les différentes neurotrophines n'est pas absolue. Cela est particulièrement vrai dans le système nerveux central, comme le montre l'absence de perturbations majeures chez les trois types d'animaux décrits ci-dessus, ou encore le fait que l'invalidation des gènes des neurotrophines se traduise par des déficits moins sévères que celle des gènes de leurs récepteurs.

Une réponse à ce dernier problème viendra sans doute de l'association d'invalidations géniques multiples. Un premier exemple vient d'être donné simultanément par deux équipes, avec une invalidation couplée des gènes de la NT -4 et du BDNF $[8,9]$. Seuls les animaux hétérozygotes survivent à cette double suppression. Les homozygotes meurent dans les 48 heures qui suivent la naissance, alors que les homozygotes porteurs d'invalidation de l'un ou de l'autre gène se développent jusqu'à l'âge adulte. En comparant les déficits observés chez les mutants invalidés "simples" et chez les souris $B D N F / N T 4(-/-)$, les auteurs révèlent que, dans le système nerveux périphérique, les neurones des ganglions sensoriels vestibulaires et trigéminés requièrent uniquement le BDNF pour leur survie, alors que les ganglions noueux et pétreux contiennent un mélange de populations sensibles à l'une, à l'autre, ou aux deux molécules. Dans le système nerveux central, les motoneurones, spinaux ou à l'origine des nerfs crâniens, ne sont pas affectés chez les animaux $B D N F / N T 4$ (-/-). Comme le nombre de ces motoneurones est significativement diminué chez les souris trkB (-/-), ces résultats montrent, pour la première fois in vivo, qu'un troisième ligand de ce récepteur, probablement la NT-3, est capable d'activer TrkB lorsque les ligands préférentiels font défaut.

La somme de ces travaux réalisés au cours des toutes dernières années montre que, en répondant au manque de possibilités d'approche directe in vivo, l'utilisation des techniques de génétique moléculaire peut permettre d'attribuer à ces différents facteurs leurs rôles respectifs dans la physiologie nerveuse

$\mathrm{m} / \mathrm{s} n^{\circ} 8$, vol.11, août 95

\section{RÉFÉRENCES}

1. Lindsay RM, Wiegand SJ, Altar A, DiStefano PS. Neurotrophic factors: from molecules to man. Trends Genet $1994 ; 17$ : 182-92.

2. Barbacid M. The Trk family of neurotrophin receptors. J Neurobiol $1994 ; 25: 1386$ 403.

3. Lamballe $F$. Les récepteurs tyrosine kinases Trk : récepteurs de forte affinité des neurotrophines. médecine/sciences $1995 ; 11$ : 1071-80.

4. Barde YA, Edgar D, Thoenen H. Purification of a new neurotrophic factor from mammalian brain. EMBOJ $1982 ; 1$ : 549-53.

5. Acheson A, Conover JC, Fandl JP, DeChiara TM, Russell M, Thadani A, Squinto SP, Yancopoulos GD, Lindsay RM. A BDNF autocrine loop in adult sensory neurons prevents cell death. Nature $1995 ; 374$. 450-3.

6. Levi-Montalcini $R$. The nerve growth factor 35 years later. Science $1987 ; 237: 1154$ 62.

7. Davies AM. The role of neurotrophins in the developing nervous system. $J$ Neurobiol $1994 ; 25: 133448$.

8. Conover JC, Erickson JT, Katz DM, et al, (16 auteurs). Neuronal deficits, not involving motor neurons, in mice lacking BDNF and/or NT-4. Nature $1995 ; 375: 235-8$.

9. Liu X, Emfors P, Wu H, Jaenisch R. Sensory but not motor neuron deficits in mice lacking NT4 and BDNF. Nature $1995 ; 375$ : 238-41.

\section{Brigitte Onteniente}

Inserm U. 421, 8, rue du Général-Sarrail 94010 Créteil Cedex, France.

\section{TIRÉS À PART}

B. Onteniente 